\title{
La gouvernance locale, une forme de développement local et durable? Une illustration par les pays
}

\section{Damien Talbot}

\section{OpenEdition}

1 Journals

\section{Édition électronique}

URL : http://journals.openedition.org/developpementdurable/2666

DOI : 10.4000/developpementdurable.2666

ISSN : $1772-9971$

Éditeur

Association DD\&T

\section{Référence électronique}

Damien Talbot, «La gouvernance locale, une forme de développement local et durable? Une illustration par les pays ", Développement durable et territoires [En ligne], Dossier 7 | 2006, mis en ligne le 29 avril 2006, consulté le 21 décembre 2020. URL : http://journals.openedition.org/ developpementdurable/2666 ; DOI : https://doi.org/10.4000/developpementdurable.2666

Ce document a été généré automatiquement le 21 décembre 2020.

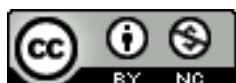

Développement Durable et Territoires est mis à disposition selon les termes de la licence Creative Commons Attribution - Pas d'Utilisation Commerciale 4.0 International. 


\title{
La gouvernance locale, une forme de développement local et durable? Une illustration par les pays
}

\author{
Damien Talbot
}

1 De façon minimaliste, le développement durable s'entend comme un mode de développement qui répond aux besoins des acteurs présents sans compromettre la capacité des générations suivantes à répondre aux leurs (Commission Mondiale sur l'Environnement et le Développement, 1987). Les principes de prévention, de précaution et de participation des acteurs sont au cœur d'un mode de développement qui se veut solidaire et partagé. La dimension temporelle est au centre de la définition du développement durable, tandis que la dimension spatiale est le plus souvent appréhendée à travers les interdépendances de voisinage (diffusion d'une pollution par exemple), ou encore dans le sens d'une déclinaison locale d'un concept global de développement durable. Dans ce cas, l'espace réduit au seul contexte physique est postulé, compris comme une dimension supplémentaire à intégrer au développement durable et finalement nié dans sa diversité. Plus rarement, les analyses cherchent à expliquer, non pas comment adapter le développement durable aux espaces locaux donnés a priori, mais de façon inverse en quoi des relations fondées sur une proximité géographique entre des acteurs sont elles-mêmes durables ou non. C'est l'objet de cet article que d'inverser le sens de l'analyse, que d'affirmer que l'espace, comme construit social, n'est pas un simple réceptacle de gouvernances autonomes mais peut au contraire contribuer (ou non) à ces mêmes gouvernances qualifiées alors de locales, certaines pouvant en outre prendre des formes durables.

2 Pour justifier cette affirmation, nous proposons en préalable de faire appel à la notion de proximité1. L'association de la proximité géographique à deux autres formes de proximité, institutionnelle et organisationnelle, permet à notre sens de proposer une définition de la gouvernance locale. La mobilisation de ce dernier concept, s'il souffre d'une certaine polysémie, présente un certain nombre d'avantages. Bertrand et Moquay 
(2004) expliquent en effet que la gouvernance, en sciences économiques, est tour à tour définie dans les approches contractuelles comme une structure justement contractuelle et complémentaire au marché qui permet de se coordonner et, dans la théorie de la régulation, comme une structure politique représentée essentiellement par l'Etat. En science politique, la gouvernance renvoie au système de décision publique préconisant une diminution de l'intervention étatique et/ou une affirmation de la participation des acteurs privés à la définition et à la mise en œuvre de l'action publique. Malgré cette polysémie, elle présente un triple avantage : premièrement, elle permet de réintroduire dans l'analyse le pouvoir, ce «banni récalcitrant » cher à Perroux (1973), à travers les conflits; deuxièmement, elle autorise l'endogénéisation de l'espace entendu comme un construit social et non un simple réceptacle des relations économiques; troisièmement, elle renouvelle le débat entre Etat, marché et société civile à travers par exemple la question de la démocratie participative (Baron, 2003). Par ce dernier point au moins, elle rejoint les problématiques de développement durable, soulignant les préoccupations communes portées par les deux notions et par là même l'intérêt de les rapprocher.

3 Les pays nous semblent constituer une illustration pertinente d'une gouvernance locale susceptible de présenter un caractère durable, puisque le pays « exprime le projet commun de développement durable du territoire (...)» (art. 25 de la Loi d'Orientation pour l'Aménagement et du Développement Durable du Territoire adoptée le 29 juin 1999). Il faut voir dans le pays un espace de projet, une instance d'animation, de concertation et de coordination prévue pour programmer et définir les priorités collectives, un outil institutionnel destiné à prendre la forme d'une gouvernance locale en mobilisant les avantages supposés de la proximité géographique. Ces pays présentent un caractère durable car, comme exemple d'ordre social localisé, ils mobilisent des initiatives locales et constituent un cadre de mise en œuvre des nouvelles modalités d'action publique. Il s'agit, par exemple, de renforcer la démocratie participative en stimulant les initiatives locales. Sur cette base volontaire, ils fédèrent notamment des communes, des syndicats professionnels, des associations autour de questions (environnementales par exemple) souvent délaissées par les collectivités locales et, si possible, dans le respect des intérêts de chacun. Au-delà de ce seul principe d'équité, le principe d'interdépendance entre les espaces, les générations et les disciplines qui aide à définir le développement durable (Laganier, et al., 2004) trouve lui aussi une illustration dans le cas du pays. La démarche des pays, décision nationale déclinée localement, suppose l'intégration du passé du territoire tout en mobilisant une grande variété d'acteurs. Autant d'éléments susceptibles de départager les caractères durables et non durables d'une gouvernance locale.

Notre propos se structure en trois temps. Le rôle ambigu de la proximité géographique sera tout d'abord souligné, considérant que si elle facilite la coordination, elle peut aussi être source de conflits qui freinent celle-ci. Une définition de la gouvernance locale sera ensuite proposée, en lui attribuant une dimension institutionnelle et une dimension organisationnelle, tout en insistant sur le rôle du pouvoir par trop souvent occulté. Enfin, grâce à une illustration par les pays, nous soulignerons quelques caractères durables et non durables de la gouvernance locale.

$5 \quad$ L'idée selon laquelle les relations économiques et les repères qui les régissent sont situés dans le temps fait l'objet d'un large consensus. Il est aussi admis que ces relations et repères sont, de la même façon, situés dans l'espace géographique. Ce dernier occupe toutefois une place marginale dans l'analyse économique contemporaine, donnant l'impression que les activités économiques se déroulent dans un monde a-spatial. Nous 
cherchons ici à dépasser cette limite, considérant que le lieu où prend place la gouvernance compte autant que le moment durant lequel elle se déroule.

6 L'espace géographique ne doit pas uniquement s'entendre comme un contexte purement physique doté d'attributs matériels dans lequel se déroulent les relations économiques. Cet espace possède une dimension sociale fondatrice qui permet de le saisir plus comme une construction active de relations que comme un « réceptacle neutre et uniforme » des stratégies des acteurs (Dupuy et Gilly, 1996). Nous nous situons dans une « conception d'un espace physique, construit, travaillé, modelé, partagé par les hommes. (...) Les espaces construits sont des transformations de l'espace physique préexistant, conservant comme fondement le cadre matériel des interactions et des échanges" (Grossetti, 1997, p. 3). L'espace est un objet de mémoire composé du nom de cet espace, de ses limites physiques, de son histoire, de son patrimoine, de modes de vie, de personnes, des coordinations précédentes, etc. Cet objet de mémoire constitue dès lors un référent cognitif, un ensemble de références communes, l'espace intervenant dans le processus de construction (destruction) des identités dans le sens où il est une composante essentielle du rapport aux autres (Pecqueur, 1997) puisque les acteurs locaux se le représentent et le font exister au regard des autres (Laganier, et al., 2004). Comme émanation d'une logique de l'action collective, la gouvernance locale repose bien sur une «transformation des propriétés de l'espace banal» (Dufourt, 1996), chaque espace devenant alors singulier puisque porteur d'une identité propre.

7 Sur la base de cette conception élargie de l'espace, nous soulignons que le partage d'une identité facilite alors les échanges et catalyse les interactions sociales (Vant, 1998). Les relations socio-économiques, encastrées dans des relations localisées de nature privée, peuvent alors être à la fois plus nombreuses et plus approfondies, sans pour autant être garanties.

8 Sur le plan institutionnel en effet, ce lien social identitaire, principalement de nature tacite et qui facilite les interactions sociales (Rallet et Torre, 2004), peut se révéler fondateur d'une confiance entre les acteurs locaux et plus généralement de ressources cognitives spécifiques qui, une fois combinées, aident à la résolution de problèmes (Colletis et Pecqueur, 1995, 2004²). Ces ressources sont toujours des résultats fragiles et transitoires, issus des coordinations antérieures. On insiste ici sur la dimension historique de la relation, largement inscrite dans la notion de proximité : l'histoire des liens compte.

9 Sur le plan organisationnel cette fois, le partage d'un même espace facilite le développement de la gouvernance. Nous renvoyons ici à l'idée classique selon laquelle l'espace physique est structuré par des infrastructures de transport et de communication, constituant un cadre matériel favorisant la circulation des informations, des biens physiques et des individus. En particulier, des acteurs ayant pour objectif la production de connaissances vont profiter d'une localisation commune dans un tel espace structuré pour satisfaire leur besoin, au moins temporaire, de face à face.

10 N'oublions pas que la proximité géographique qui lie certains acteurs est d'abord subie et peut se transformer en une contrainte, tant on voit mal les membres d'une association, d'un syndicat professionnel ou encore les élus se délocaliser pour se soustraire à un voisinage indésirable. Ainsi que le soulignent Rallet et Torre (2004), être voisin peut signifier s'ignorer, d'autant que les technologies de la communication offrent dorénavant la possibilité d'être présent ici et ailleurs, et donc d'être "proche» de quelqu'un géographiquement éloigné. Cela peut aussi signifier se détester car, si la proximité géographique autorise une meilleure connaissance de l'autre du fait du partage d'une 
identité minimale, cette connaissance peut conduire tout autant les acteurs à favoriser des relations proches qu'à les rejeter, en fonction de la mémoire des coordinations réussies ou échouées. De façon intermédiaire, le voisinage peut aussi engendrer la méfiance. Ainsi, dans le cas d'une coordination réunissant une multiplicité d'acteurs, il peut exister des conflits latents résultant de relations passées entre une partie des participants. Dans la première situation, les ressources spécifiques cognitives sont inexistantes; dans la deuxième et la troisième, ces mêmes ressources auront un impact négatif sur la relation. C'est notamment le cas lorsque l'objectif de la gouvernance locale est de permettre une utilisation raisonnée d'une ressource consommée conjointement entre les usagers d'un même lieu (pensons à l'eau), après une période entachée par des conflits de voisinage au sujet de problèmes de répartition, de pollutions diffuses, etc., qui auraient affecté la ressource. La littérature sur les conflits d'usage de l'espace (cf. par exemple Caron et Torre, 2004; Kirat et Lefranc, 2004; Lahaye, 2004) cite nombre d'exemples d'inégalités liées à la situation topographique des acteurs (un usager subit la pollution de l'eau provenant d'un autre usager situé en amont). La proximité géographique peut donc être constitutive d'inégalités fondamentales créant des rapports de pouvoir entre les acteurs d'une gouvernance locale. Ce n'est pas pour autant que toutes les interactions deviennent définitivement impossibles, car les acteurs qui n'ont pas la capacité de se relocaliser ailleurs doivent faire face à cette externalité négative de proximité, selon les termes de Rallet et Torre (2004). Cet état de fait constitue à lui seul une forte incitation au compromis, et plus généralement à la coordination en mobilisant la dimension positive des ressources cognitives communes, c'est-à-dire le lien social identitaire, obligés que sont les acteurs de s'entendre in fine.

11 Finalement, la proximité géographique agit sur la coordination, certes en la facilitant, mais aussi en la contraignant: elle est tout à la fois un potentiel et une contrainte (Pecqueur et Zimmermann, 2004). Reste à caractériser cette coordination : pour ce faire, nous faisons appel aux propriétés relationnelles de la proximité, dite alors organisée, qui présente à nos yeux une double dimension, institutionnelle et organisationnelle. Et c'est l'association des diverses formes de proximités qui définit à selon nous la gouvernance locale.

12 Juillet (2000) propose de distinguer, au sein de la coordination, la conceptualisation du monde réel (le paradigme) des manifestations concrètes de patterns sociaux et organisationnels institués (les modes de gouvernance). Dans cette perspective, nous suggérons de caractériser la proximité organisée par la distinction de deux niveaux analytiques intimement liés : premièrement, elle se compose de repères nécessaires à toute coordination (niveau institutionnel), deuxièmement des formes mêmes de cette coordination (niveau organisationnel). Autrement dit, nous déclinons la proximité en trois dimensions: géographique, institutionnelle, organisationnelle. Un débat s'est d'ailleurs fait jour entre les auteurs de la proximité, un premier courant qualifié d'interactionniste (Pecqueur et Zimmermann, 2004), se limitant à deux formes de proximité (géographique, organisée), un second, d'inspiration institutionnaliste (Kirat et Lung, 1995 ou encore Gilly et al. 2004), déclinant la proximité en trois composantes (géographique, institutionnelle, organisationnelle) (Gilly et Lung, 2004). Le premier courant, actuellement dominant au sein du groupe de chercheurs «Dynamiques de proximité ", présente l'inconvénient à nos yeux de rendre moins aisée l'intégration dans l'analyse (sans être toutefois occultée) de l'influence des objets sociaux collectifs que sont par exemple les repères communs ou plus généralement les institutions. Ou plutôt, en ne 
les intégrant pas d'emblée, le risque est plus important de sous-estimer leur rôle. De même que les chercheurs du groupe se sont rassemblés autour de la nécessité d'endogénéiser l'espace dans la théorie économique, nous plaidons pour une endogénéisation des institutions (règles collectives, représentations communes, repères partagés, etc.) à l'instar des travaux institutionnalistes sur la gouvernance. Ainsi, définir la gouvernance comme une combinaison de proximité nécessite d'accorder une large place à la dimension institutionnelle de cette dernière.

13 La gouvernance est en effet conditionnée par l'existence d'un partage minimum de repères que peut constituer par exemple un projet commun, constitutif d'une proximité institutionnelle entendue comme une condition certes contraignante, mais aussi permissive à la coordination. La réalisation concrète d'un projet commun implique, quant à elle, des actions collectives, une activité commune finalisée, bref une proximité organisationnelle. Revenons sur ces points.

Dans une perspective institutionnaliste, les repères d'une coordination localisée apparaissent comme des modèles normatifs qui définissent ce qui semble devoir être fait dans un environnement économique partiellement connu et compris (Hodgson, 1998). Ces repères sont à la fois des contraintes et des ressources pour l'action (Corei, 1995; Hodgson, 1996). Le premier aspect renvoie au contrôle de l'action, le second à sa libération (Commons, 1934). Ce contrôle se réalise par :

- - d'une part, la détermination du champ des possibles. Les repères, à l'instar des règles qui présentent la particularité d'être formulées et acceptées (Renault, 1995) par les acteurs locaux qui les appliquent, vont définir les devoirs, les libertés et les droits de chacun, précisant donc ce qu'il est possible de faire et ce qui ne l'est pas. Mais il ne faut pas voir dans ces règles une proscription ou une prescription précise des comportements : les règles guident l'action sans la produire, ce sont en quelque sorte des « théories » de l'action. Elles nécessitent donc une interprétation, c'est-à-dire un accord (un compromis) sur le contenu de l'activité normale (Reynaud, 1994). Ici, l'existence d'une mémoire de coordinations réussies créatrice de confiance peut faciliter le passage d'un compromis, comme autant de ressources cognitives spécifiques accessibles aux acteurs de la gouvernance locale ;

- - et d'autre part, la régulation des conflits. Ces derniers étant temporairement prévenus, ils ne peuvent plus ruiner la sécurité des anticipations nécessaire à l'action collective dans un monde incertain (Bazzoli et Kirat, 1996). L'efficacité de ce contrôle résulte des sanctions qui lui sont associées, ou plus précisément des anticipations que font les acteurs de ces sanctions (Commons, 1934). Un exemple de sanction peut consister en l'éviction d'un acteur d'un projet porté par la gouvernance locale.

La libération de l'action est rendue possible par un double phénomène de réduction de l'incertitude et d'homogénéisation des comportements, les deux processus s'entrainant mutuellement. En effet :

- - les repères sont subjectifs, ils sont donc porteurs de sens, de valeurs, voire d'engagements réduisant l'incertitude inhérente à toute action. Il s'agit là d'une vision positive de leur finalité, les acteurs de la gouvernance les mobilisant non seulement pour satisfaire leur intérêt, mais aussi parce qu'ils leur semblent justes. Ici, les principes d'équité ou d'interdépendance qui régissent le développement durable ( $c f$. ci-après) sont des illustrations de repères perçus comme justes ;

- - les repères établissent des modèles : en bornant les comportements, ils rendent ces derniers plus semblables et donc plus prévisibles même si, nous l'avons vu, des problèmes d'interprétation peuvent se poser. 
16 Ainsi, dans un monde moins incertain où, d'une part, les conflits sont transitoirement régulés par l'existence de repères, et où, d'autre part, l'espace ouvert par ces mêmes repères et par leur interprétation autorise une action commune, le passage de divers compromis fondant la gouvernance locale devient alors possible. Ces accords institutionnels portent sur les repères, à l'instar des règles et des principes du développement durable, qui se doivent d'être partagés au moins temporairement pour engager une action collective.

Ce partage suppose la mise en œuvre d'un apprentissage institutionnel. Il s'agit de réduire les blocages dans le processus d'apprentissage collectif en modérant la dissonance cognitive, ce qui suppose de se doter d'un même cadre de référence, d'une vision similaire du monde (Marengo, 1995) afin de rendre possible la coordination collective. Cremer (1990) rappelle que trois types de connaissances acquises, comme autant de repères, aident à la coordination : une connaissance commune des faits, une connaissance commune des règles et une connaissance commune d'un langage. Ces repères collectifs, en contrôlant et en libérant l'action, vont permettre aux agents d'apprendre à se coordonner, avant d'apprendre ensemble, ce qui renforce l'efficience et la cohérence de la gouvernance locale. Les repères, une fois appris, facilitent les apprentissages futurs car ils constituent des structures de cognition et de comportement indispensables pour l'acquisition d'outils pratiques et intellectuels (Hodgson, 1997). Ces processus d'assimilation donnent en outre aux repères de la gouvernance locale un caractère partagé qui fonde l'appartenance à celle-ci. L'apprentissage institutionnel devient finalement un processus de généralisation et de transmission des repères aux participants à la gouvernance, constitutif d'une proximité institutionnelle. Il est supposé être intense, tant la gouvernance locale suppose le partage, toujours plus ou moins équitable, des ressources cognitives spécifiques.

18 C'est dans ce cadre que vont s'exprimer les intentionnalités des individus. L'individu conserve en effet une liberté d'action face aux influences issues du contexte institutionnel, liberté qui s'exprime notamment dans l'intention. Dès lors, le mode de gouvernance est une expression collective de la volonté des acteurs de la gouvernance, un consentement issu de processus de conflits et de compromis, un arrangement sur les objectifs de l'action collective et sur les actions à mener afin de les réaliser. Ces actions collectives intentionnelles et délibératives, constitutives d'une proximité organisationnelle, sont orientées vers des objectifs communs (résoudre un problème productif, construire un projet de développement par exemple) et autorisées temporairement par l'existence de divers compromis collectifs, par nature instables, entre des intérêts divergents. Notamment, elles prennent la forme d'une acquisition de compétences et de création de connaissances communes, c'est-à-dire d'un apprentissage, organisationnel cette fois ${ }^{3}$.

19 Ainsi, la gouvernance locale, comme combinaison de proximités, devient le vecteur d'une cohérence singulière levant provisoirement et partiellement l'incertitude inhérente à toute action collective par la réduction et non l'évacuation des conflits (Gilly et al., 2004). Structurées par et pour la création et la mise en œuvre d'un projet commun, ces interactions sont enchâssées dans des rapports de pouvoir puisque les ressources matérielles et cognitives sont inégalement distribuées entre des acteurs économiques, politiques et sociaux aux intérêts variés. L'enjeu est de mettre en place une coordination efficace dans un contexte de multiplicité des sources d'autorité et de pouvoir (Juillet, et al., 2001). 
20 Si l'on envisage le pouvoir comme une relation (Perroux, 1973), il apparaît alors comme un processus interactif se développant sur deux niveaux : au niveau institutionnel par les représentations du statut social de chaque agent comprises comme des repères dans la coordination, et au niveau organisationnel par sa dimension stratégique (Bernard et al., 1997).

21 La compréhension de la première dimension du pouvoir nécessite d'intégrer le fait que les acteurs n'émergent pas ex nihilo afin de se coordonner, mais qu'ils sont situés au sein d'espaces de coordination à l'instar de la gouvernance, qui sont autant d'espaces de pouvoir. Cette dimension institutionnelle du pouvoir s'inscrit dans une problématique de légitimité. En effet, même si un acteur a le sentiment de subir ce pouvoir, le pouvoir n'existe que par le consentement tacite ou explicite de celui-ci (Huisman et Ribes, 1994), consentement qui suppose que le pouvoir soit reconnu comme légitime ${ }^{4}$.

Cette légitimité concerne simultanément trois objets interconnectés : premièrement, un pouvoir est reconnu comme légitime s'il est exercé par le ou les agents légitimes; deuxièmement le pouvoir doit s'exercer dans le cadre institutionnel de la gouvernance, c'est-à-dire dans l'espace ouvert par les repères appris et acceptés par les participants ; troisièmement, le pouvoir s'exerce pour atteindre un ensemble d'objectifs, un projet (Reynaud, 1993). En ce sens, la légitimité renvoie à un problème d'efficacité en fonction de critères retenus par chacun des agents participant à la gouvernance. Le pouvoir est nécessairement performatif (Huisman et Ribes, 1994) se manifestant par une action recherchant la réussite. Et cette réussite confère une légitimité, qui elle-même autorise l'exercice du pouvoir dans la gouvernance locale.

Cet exercice du pouvoir, qui relève du niveau organisationnel de la gouvernance locale, peut se définir à travers quatre traits distincts. Premièrement, le pouvoir est une relation, et non pas un attribut des acteurs; deuxièmement, cette relation est déséquilibrée dans la mesure où il y a une asymétrie de ressources cognitives et matérielles entre les agents face à une situation, ce qui rend les termes de l'échange plus favorables à l'une des parties; troisièmement, l'incertitude est une composante centrale de la relation de pouvoir, l'imprévisibilité potentielle du comportement des agents dépendant du degré d'autonomie dont ils disposent; enfin et quatrièmement, la dimension relationnelle du pouvoir suppose une intentionnalité dans la coordination. Concrètement, un agent peut légitimement exercer une domination en imposant ses propres règles et objectifs à l'ensemble des participants à la gouvernance. Toutefois, la gouvernance se caractérise par l'absence d'un acteur central de type hégélien, à l'instar de l'Etat qui devient dans notre cadre un acteur moins régulateur que médiateur. Cette absence justifie d'ailleurs le recours à ce nouveau terme pour rendre compte de l'émergence de coordinations dans lesquelles le pouvoir est plus une affaire de réseaux que de hiérarchie (Baron, 2003). La notion de gouvernance permet donc, en décrivant une nouvelle organisation du pouvoir typique d'une démocratie participative, de le réintroduire. Cette particularité, parmi d'autres, est d'ailleurs souvent invoquée pour attribuer à la gouvernance locale des propriétés de durabilité.

24 La définition de la gouvernance locale comme une combinaison de proximités a permis de mettre à jour certaines caractéristiques de durabilité qu'il nous faut maintenant expliciter, en illustrant notre propos par les pays. Nous revenons en préalable sur les principes de développement durable regroupés sous le vocable de durabilité. 

développement économique et social sont définis en fonction de l'intérêt commun et de la durée, afin de modifier le contenu de la croissance (Commission Mondiale sur l'Environnement et le Développement, 1987). Ainsi, la notion de développement durable présente le mérite de redonner au temps de la profondeur, et par là même de rappeler les contraintes du temps long et donc la nécessité d'intégrer les préoccupations économiques et écologiques dans la prise de décisions. Cette caractéristique du temps est souvent absente des réflexions des acteurs socio-économiques pour lui préférer, face à l'urgence des problèmes actuels, des réponses rapides mais finalement peu durables, voire éphémères. Toutefois, la notion de durabilité ne peut se réduire à la pérennité. Il faut y associer les principes de participation, de solidarité, de prévention/précaution, de santé et de qualité de vie, d'accès au savoir, d'économies des ressources, d'efficacité économique, etc., la déclaration de Rio en énumérant au total 27.

De façon plus précise mais réductrice, deux principes définissent le développement durable: interdépendance et équité (Laganier et al., 2004). L'interdépendance est premièrement temporelle puisque les effets d'une action sont durables, deuxièmement intersectorielle car les actions dans un secteur de l'économie ont des effets secondaires dans d'autres secteurs, troisièmement interdisciplinaire car les activités humaines comme l'économie, le social, la politique, l'écologie, etc., se façonnent les unes les autres, et quatrièmement spatiale car une action sur un lieu induit des effets de débordement sur d'autres espaces. L'interdépendance n'est donc pas un simple phénomène local et pose notamment les questions de la concurrence versus coopération des territoires et des rapports local / global. L'équité doit, quant à elle, caractériser autant que faire se peut, les liens intergénérationnels, notre développement ne devant pas hypothéquer celui des générations à venir, mais aussi fonder les liens intragénérationnels, notre développement ne devant pas se réaliser au détriment de certains de nos contemporains. Dans ce dernier cas, le respect du principe d'équité induit de nouvelles formes d'expression du débat public (Beaurain, 2004), que l'on songe par exemple à la démocratie participative qui autorise la participation effective des citoyens à la prise de décisions. De même, les politiques de développement doivent prendre en considération les problèmes d'accès aux ressources et de distribution des coûts et des avantages, assurer dans la mesure du possible l'égalité des chances pour tous. La question est maintenant de savoir en quoi la gouvernance locale, lorsqu'elle prend la forme d'un pays, est porteuse de tels principes.

En endogénéisant l'espace, la gouvernance locale autorise l'introduction de la proximité géographique comme contrainte dans l'analyse. Par le rappel du caractère irréversible de la localisation de certains acteurs, la démarche de pays souligne de la sorte l'interdépendance spatiale entre les acteurs locaux. Elle cherche d'ailleurs à accentuer ce principe en insistant sur les interactions entre villes et espaces ruraux. Considérant que les espaces ruraux ne disposent que rarement des ressorts de la croissance interne, cette démarche prend en compte les effets de polarisation et d'agglomération qui caractérisent les centres urbains (Aubert et al., 2004). Cette interdépendance spatiale concerne aussi l'articulation des espaces locaux et globaux. Si parler de gouvernance locale permet en effet de souligner la relative autonomie des processus locaux de développement (Bertrand et Moquay, 2004), cela ne signifie pas que le niveau global ${ }^{5}$ est oublié et que les causalités entre les deux niveaux sont occultées. Les acteurs sont aussi insérés dans des réseaux a-territoriaux, en relation avec des acteurs éloignés (Gilly et Wallet, 2004). Ils mobilisent simultanément dans leur coordination diverses échelles spatiales. Autrement 
dit, il existe une causalité circulaire entre le local et le global, une influence mutuelle qui souligne l'interdépendance entre les échelles spatiales.

Les pays illustrent bien ce cas de repères et de pratiques de gouvernance locale, définies et impulsées à l'échelon national pour être déclinées localement de façon plus ou moins diverse. Ainsi, la politique des pays est l'expression d'une nouvelle représentation du développement local qui se fait jour dans la pensée publique française. Cette création des pays renvoie à la nécessité de repenser les interventions publiques, cherchant à simplifier les procédures de décisions et les actions, dans un contexte de politiques européennes s'appuyant sur un principe de subsidiarité. Dorénavant, le développement local se veut le résultat d'un partenariat entre acteurs locaux en associant les aspects économiques, sociaux et culturels et en rompant avec une politique d'aménagement fondée sur une logique purement redistributrice des richesses nationales sous formes d'aides et de primes diverses. Fondamentalement, les richesses ne sont plus allouées par l'Etat, mais doivent être créées localement avec le concours de l'Etat. Le territoire ne doit plus être seulement aménagé mais d'abord développé (Bernard, 2001). On le voit, les représentations du développement local, comme autant de repères, ont profondément évolué notamment sous l'influence de l'Union Européenne, partenaire avec lequel l'Etat doit composer tant il est devenu incontournable dans le financement des projets.

Cette dynamique institutionnelle, au-delà des seules transformations des représentations, a conduit à la création de règles juridiques régissant l'organisation des pays. Ce dernier est une notion instituée par la Loi d'Orientation pour l'Aménagement et le Développement du Territoire du 4 février 1995 (dite aussi Loi Pasqua), reprise par la Loi d'Orientation pour l'Aménagement et le Développement Durable du Territoire adoptée le 29 juin 1999 (dite aussi Loi Voynet), et amendée par la Loi « Urbanisme et Habitat » du 2 juillet 2003 qui apporte quelques changements dans la procédure de constitution et dans la gestion des projets de pays. Selon la Loi Pasqua, le pays manifeste la communauté d'intérêts socio-économiques. Le pays n'est ni une nouvelle circonscription privée de toute vie juridique autonome, ni une nouvelle collectivité agissant en nom propre : sur un plan juridique, il se définit comme un groupement territorial d'aménagement et de développement local possédant sa personnalité sans détenir la gestion de ses propres affaires, le situant entre la circonscription et la collectivité. Le but est de réorganiser les services de l'Etat, en établissant comme règle, la primauté des projets sur la structure et en autorisant les acteurs locaux à s'organiser en dehors des périmètres administratifs et dans un cadre commun cohérent (Gilly et Wallet, 2004). Cet espace de coordination cherche à réunir de façon peu contraignante le territoire institutionnel, celui de l'action publique, avec le territoire économique, celui des acteurs socio-économiques.

Plus de 300 démarches de pays ont été constatées à ce jour ${ }^{6}$, dont plus de 80 ont déjà abouti à une reconnaissance de l'Etat. Au sein de cette variété ${ }^{7}$, certains pays mobilisent une méthodologie de développement durable ${ }^{8}$, de façon plus ou moins explicite. Cette mobilisation est parfois clairement affirmée car la Loi Voynet prévoit qu'une charte de pays puisse faire appel à un Agenda 21 local, compris comme l'instrument politique visant à décliner localement les grands principes de l'Agenda 21 établi au Sommet de Rio en 1992. L'Agenda 21 local est en effet un projet de développement durable du territoire misant sur la recherche de convergences entre performances économiques, sociales et environnementales. Ce projet se traduit en programmes d'actions définissant les objectifs et les moyens de mise en œuvre du développement durable du territoire, l'ensemble étant évalué régulièrement selon une grille d'indicateurs et en concertation avec les parties 
concernées. L'Agenda 21 local désigne tout autant la dynamique d'élaboration concertée que le document concret traduisant le programme d'actions. Clairement, il souligne la vocation de l'échelon local à devenir l'un des espaces pertinents du développement durable, en introduisant les principes de durabilité au sein même du projet collectif porté par le pays.

31 L'interdépendance intergénérationnelle est intégrée dans la démarche des pays puisque la charte, qui exprime le projet commun, doit tenir compte du passé du territoire et proposer une réflexion prospective. La loi prévoit ainsi le temps nécessaire à la maturation et à la mise en œuvre de la charte (Aubert et al., 2004), les projets se devant d'apporter des solutions durables aux problèmes de gestion de ressources par exemple.

Le principe d'interdépendance entre acteurs, contemporains cette fois, est aussi respecté du fait de la variété des acteurs du développement local : entreprises, collectivités publiques, associations, syndicats, simples citoyens, etc. Les coordinations se font plus ouvertes, l'étanchéité des catégories d'acteurs faisant place à une porosité grandissante. La notion de gouvernance elle-même, par nature à la confluence de la science économique et de la science politique, dénote encore l'interdisciplinarité. L'une des conséquences attendues de cette dernière est l'accroissement de la cohésion sociale, le renforcement de solidarités locales, par la concertation entre acteurs hétérogènes.

Le principe éthique trouve ici sa traduction dans la démocratie participative qui doit caractériser le fonctionnement des pays. L'introduction de la notion de gouvernance locale a permis de souligner que la proximité géographique ne peut suffire à elle seule pour impulser une relation, qu'il faut y associer l'intentionnalité des acteurs s'exprimant par exemple dans la réalisation d'un projet commun. Il s'agit de passer de l'existence d'une identité locale, portée par la proximité géographique, à la définition d'un intérêt commun aux acteurs proches, porté par la proximité institutionnelle, à l'origine d'actions collectives constitutives d'une proximité organisationnelle, l'ensemble définissant la gouvernance locale. La causalité inverse est aussi vraie puisque la définition de l'intérêt commun peut être favorisée par l'instauration d'un mode de gouvernance fondé sur la démocratie participative. Cette démocratie (parfois qualifiée de " démocratie de proximité ») est un outil de résolution de problèmes locaux par la création de compromis. Cette pratique est une modalité de gestion de la tension permanente entre intérêts individuels et compromis collectif, en associant les bénéficiaires d'un projet à sa définition. On suppose alors que le programme défini localement sera mieux compris (et donc légitimé) car il est censé mieux répondre aux besoins des bénéficiaires. Le principe de démocratie participative ouvre la voie aux initiatives individuelles, autorise l'expression des intentionnalités des individus dans une démarche avant tout collective.

34 Finalement, par certains aspects au moins, les pays mettent en œuvre les principes du développement durable. Toutefois, la réalité montre, au-delà de toute vision idéalisée et des affirmations volontaristes, qu'ils rencontrent dans leur fonctionnement de nombreuses difficultés qui limitent leur durabilité.

35 Le premier écueil que rencontrent actuellement les pays concerne l'épineuse question de la définition du périmètre. Trop souvent les propositions de construction de pays ne recouvrent pas des périmètres pertinents et peuvent, par exemple, renvoyer à une circonscription électorale, satisfaisant par là même les intérêts d'un seul individu et non les principes du développement durable. Ces découpages ne permettent pas de fonder le pays sur un espace vécu, de mobiliser une identité partagée. Comme il est affirmé dans les Lois Pasqua et Voynet, les pays se doivent de présenter une certaine cohérence 
historique, géographique, économique ou sociale, bref de constituer « une petite région naturelle» (Bernard, 2001). La question de la bonne utilisation de la proximité géographique, d'une délimitation cohérente des pays avec leur histoire, est centrale afin que celle-ci puisse faciliter la coordination.

Mais une définition pertinente du périmètre du pays, si elle constitue une condition nécessaire à leur réussite, n'est pas pour autant suffisante. Nous avons vu que pour parler d'une gouvernance locale, cette proximité géographique doit être associée aux autres formes de proximités. À ce stade, au moins deux cas de figures sont possibles : soit des proximités institutionnelle et organisationnelle se sont historiquement construites localement au fil des projets communs, soit l'action publique tente de les définir globalement pour les imposer localement.

Dans le premier cas, l'outil institutionnel que constitue le pays ne vient que soutenir un processus relativement autonome et endogène au territoire. Il peut même dans certaines situations le perturber. Les obligations légales qu'impose la politique de pays peuvent en effet entrer en tension avec les mécanismes locaux de coordination en place, désorganiser une gouvernance locale déjà existante qui avait ses propres pratiques, et accroître par là même les coûts de coordination (cf. l'exemple du Pays des Coteaux de Bigorre proposé par Gilly et Wallet, 2004).

Dans le second cas, les règles juridiques définies par les lois Pasqua et Voynet constituent le socle commun qui régit les relations entre les acteurs participant à la gouvernance locale. Bertrand et Moquay (2004) notent ici une contradiction à vouloir produire du développement en appliquant une logique homogénéisante face à une diversité territoriale. Il y a un paradoxe à chercher à impulser des dynamiques locales par nature singulières à partir d'un cadre généralisé, même souple. Il faut voir là l'une des limites de certains pays car la gouvernance locale ne se décrète pas, mais se construit localement et progressivement dans le temps. Il s'agit ici de savoir si la proximité géographique a joué ou non un rôle positif dans le partage de repères et de pratiques autorisant l'émergence d'un projet collectif porté par l'outil institutionnel que constitue le pays. Autrement dit, les trois formes de proximité se recouvrent-elles pour constituer une capacité à se coordonner localement, un potentiel d'action collective? L'existence d'un tel potentiel local, qui ne peut être impulsé d'un niveau global, est un élément fondateur de la réussite des pays, outil fondé, rappelons-le, sur une logique de projet et non de structure. Il faut voir là une explication de la diversité des situations constatées empiriquement, diversité des potentiels locaux de coordination et donc diversité dans la qualité des projets.

Enfin, dans l'ensemble des situations évoquées, le principe d'interdépendance entre les acteurs pose des problèmes de fonctionnement. Cet espace de coordination qui regroupe divers acteurs aux logiques différentes (administratives, politiques, économiques), même s'il constitue une possibilité de mettre en place de nouvelles coordinations correctrices des blocages issus par exemple d'une décentralisation inachevée, devient un enjeu de pouvoir, un révélateur des conflits locaux. Les relations de pouvoir, si elles concourent à la résolution des conflits en les contrôlant, peuvent aussi les générer. La pratique démontre que c'est lorsque les conflits institutionnels et politiques sont mis entre parenthèses que les pays fonctionnent. La gouvernance locale doit être un espace de pacification temporaire, au sein duquel conflits d'appropriation et de compétences sont transitoirement mis de côté. Et ceci procède de processus de mise en confiance impossibles à décréter. Dans le même temps, l'apaisement des conflits ne doit pas 
conduire à une coordination atone: la réalité montre en effet que certains pays deviennent un lieu consensuel frôlant l'immobilisme (Bernard, 2001).

Au total, si les pays, comme illustration d'une gouvernance locale, présentent dans leur fonctionnement observable des caractères de durabilité, ce bilan doit être mitigé.

41 Cet article a débuté par une analyse des vertus de la proximité géographique tout en soulignant dans le même tmps les limites de celle-ci. Il est notamment apparu, que si elle constitue indéniablement un facilitateur de la coordination, elle peut aussi être vécue comme une contrainte par des acteurs proches qui doivent faire face à des externalités négatives. Cela souligne le fait que la proximité géographique, au-delà de l'opportunité qu'elle représente, doit être associée, pour produire du développement, à des proximités institutionnelle et organisationnelle, l'ensemble pouvant conduire à la création d'une gouvernance locale. La question est de savoir comment induire une telle association. Peut-on l'impulser à partir d'un échelon spatial supérieur à celui du territoire, comme c'est le cas dans la politique de pays, en espérant que les acteurs locaux relaieront cette impulsion? La politique des pays consiste finalement, et c'est probablement sa principale limite, à vouloir universaliser le singulier, à reproduire une gouvernance locale dont les principales règles de fonctionnement sont inscrites dans la loi, réduisant de la sorte le caractère original et spontané du développement local. Conscient de cette limite, le législateur laisse d'ailleurs une forte autonomie de gestion aux acteurs locaux. Mais plus fondamentalement, ne s'agit-il pas là d'une idéalisation du rôle de la proximité géographique, perçue comme une condition suffisante au développement d'une multitude d'espaces ? Il est probable que seule une minorité des 300 pays reconnus à ce jour seront capables de mener, de façon pérenne, un grand nombre de projets, que les coordinations sclérosées par les conflits ou par divers immobilismes, révélateurs de l'absence de proximités institutionnelle et organisationnelle, conduisent nombre de pays à l'échec. La proximité géographique reste une opportunité toujours singulière d'impulser un développement local particulier.

\section{BIBLIOGRAPHIE}

Aubert F., Lepicier D., Perrier-Cornet P., et Wavresky P., 2004, "Structure économique des territoires : une analyse des disparités micro-régionales à l'échelle des pays en France", in XLème Colloque de l'ASRDLF, Convergence et disparités régionales au sein de l'espace européen, Bruxelles, septembre.

Baron C., 2003, "La gouvernance : débats autour d'un concept polysémique", Revue Droit et Société, $\mathrm{n}^{\circ} 54$, juin.

Bazzoli L., et Kirat T., 1996, "La relation entre le droit et l'économie dans les traditions institutionnaliste et post-coasienne : enjeux pour l'analyse évolutionniste", colloque

Évolutionnisme, fondements, perspectives et réalisations, Paris Sorbonne, METIS, septembre. 
Beaurain C., 2004, "Gouvernance environnementale locale et comportements économique : un nouvel éclairage sur l'articulation du temps et de l'espace", in Revue Développement Durable et Territoires, dossier $n^{\circ} 2$ Gouvernance locale et Développement durable.

Bernard G., 2001, La politique des pays face aux systèmes locaux : le cas du Pays d'Auge, mémoire de troisième année, Institut d'Etudes Politiques de Toulouse.

Bernard P., Talbot D., Wallet F., 1997, "Pouvoirs, proximités et apprentissages, une relecture des relations par la dynamique interaction / action", colloque Proximités et coordination économique, Creuset, Gate, Lyon, mai.

Bertrand N., Moquay P., 2004, "La gouvernance locale, un retour à la proximité", Economie rurale, $\mathrm{n}^{\circ}$ 280, mars-avril, pp. 77-95.

Caron A., Torre A, 2004, "Quand la proximité devient source de tensions : confits d'usages et de voisinage dans l'espace rural", 4th Proximity Congress Proximity, Networks and Co-ordination, Marseille, june.

Colletis G., Pecqueur B., 1995, "Politiques technologiques locales et création de ressources spécifiques", in Rallet A., et Torre A., (eds), Économie Industrielle et Économie Spatiale, Economica, Paris.

Colletis G., Pecqueur B., 2004, "Révélation de ressources spécifiques et coordination située", 4th Proximity Congress Proximity, Networks and Co-ordination, Marseille, june.

Commission Mondiale sur l'Environnement et le Développement, 1987, Notre avenir à tous, traduction française 1988, Montréal, Editions du Fleuve.

Commons J.R., 1934, Institutional Economics. Its Place in Political Economy, The MacMillan Compagny, New York.

Corei T., 1995, L'économie institutionnaliste, les fondateurs, collection "Économie de poche", Economica, Paris.

Cremer J., 1990, "Common knowledge and the coordination of economic activities", in Aoki M., Gustafsson B., Williamson O. E. (eds), The Firm as a Nexus of Treaties, London Sage, London.

Dufourt D., 1996, "Le territoire comme création institutionnelle dans une conception spatiale des politiques technologiques", in Massard N. (éd.) Territoires et politiques technologiques : comparaisons régionales, collection "Villes et entreprises", L'Harmattan, Paris.

Dupuy C., Burmeister A., (eds), 2003, Entreprises et territoires, les nouveaux enjeux de la proximité, La Documentation Française, Paris.

Dupuy C., Gilly J. P., 1996, "Apprentissage organisationnel et dynamique territoriale", in Pecqueur B. (ed.), Dynamiques territoriales et mutations économiques, L'Harmattan, Paris.

Gilly J. P., Leroux I., Wallet F., 2004, "Gouvernance et proximité", in Zimmermann J.B., Pecqueur B., (eds), Economie de proximités, Paris, Hermès-Lavoisier.

Gilly J.P., Lung Y., 2004, "Proximités, secteurs, territoires", 4th Proximity Congress Proximity, Networks and Co-ordination, Marseille, june.

Gilly J. P., Wallet F., 2004, "Enchevêtrement espaces de régulation et gouvernance locale. Les processus d'innovation institutionnelle dans la politique des pays en France", in XLème Colloque de l'ASRDLF, Convergence et disparités régionales au sein de l'espace européen, Bruxelles, septembre.

Grossetti M., 1997, "Proximité spatiale et proximité relationnelle", colloque Proximités et coordination économique, Creuset, Gate, Lyon, mai. 
Hodgson G., 1996, "Corporate culture and the nature of the firm", in Groenewegen J., ed., Transaction Cost Economics and Beyond, Kluwer Academic Publishers, Boston / Dordrecht / London.

Hodgson G., 1997, "The ubiquity of habits and rules", Cambridge Journal of Economics, vol. 21, n6, pp. 663-684.

Hodgson G., 1998, "The Approach of Institutional Economics", Journal of Economic Literature, vol. $36, \mathrm{n}^{\circ} 1$, pp. $166-192$.

Huisman B., Ribes F., 1994, Les philosophes et le pouvoir, Dunod, Paris.

Johnson B., Lundvall B. A., 1992, "Closing the institutional gap ?", Revue d'Économie Industrielle, n 59, premier trimestre, pp. 111-123.

Juillet L., 2000, "Pouvoir, démocratie et gouvernance en réseaux" in Andrew C., Cardinal L. (éd.), Gouvernance et démocratie, Presses de l'Université d'Ottawa, Ottawa.

Juillet L., Paquet G., Scala F., 2001, "Gouvernance collaborative, imputabilités douces et contrats moraux : un cadre d'analyse", in Revue Gouvernance, 2:1, pp. 85-95.

Kirat T., Lefranc C., 2004, "Les conflits d'usage et leur expression territoriale : une analyse des profils contentieux de sept départements français", 4th Proximity Congress Proximity, Networks and Co-ordination, Marseille, june.

Kirat T., Lung Y., 1995, "Innovations et proximités : le territoire, lieu de déploiement des processus d'apprentissage", in Lazaric N., Monnier J. M. (coordonné par), Coordination économique et apprentissage des firmes, Economica, Paris.

Laganier R., Villalba B., Zuindeau B., 2002, "Le développement durable face au territoire : éléments pour une recherche pluridisciplinaire", in Revue Développement Durable et Territoires, dossier $n^{\circ} 1$ Approches territoriales du Développement Durable.

Lahaye N., 2004, "Les réseaux de négociation créent-ils les conditions de résolution des conflits environnementaux", 4th Proximity Congress Proximity, Networks and Co-ordination, Marseille, june.

Marengo L., 1995, "Apprentissage, compétences et coordination dans les organisations", in Lazaric N., Monnier J. M. (éd.), Coordination économique et apprentissage des firmes, Economica, Paris.

Pecqueur B., 1997, "Processus cognitifs et construction des territoires économiques", in Guilhon B., Huard P., Orillard M., Zimmermann J. B. (sous la direction de), Économie de la connaissance et des organisations, L'Harmattan, Paris.

Pecqueur B., Zimmermann J. B. (eds), 2004, Economie de Proximités, Hermès, Lavoisier, Paris.

Perroux F., 1973, Pouvoir et Économie, Dunod, Paris.

Rallet A., Torre A., 2004, "Proximité et localisation", Economie rurale, n² 280, mars-avril, pp. 25-41.

Renault M., 1995, "Communication, interactions et coordination des comportements", colloque La connaissance dans la dynamique des organisations productives, CEFI-GRACSE-GREQAM-LEST, Aixen-Provence, septembre.

Reynaud B., 1994, "De la règle à l'apprentissage de la coordination", colloque Limitations de la rationalité et constitution du collectif, Cerisy, septembre.

Reynaud J. D., 1993, Les règles du jeu. L'action collective et la régulation sociale, série Sociologie, Armand Colin, Paris.

Vant A., 1998, "Proximités et géographies", in Bellet M., Kirat T., Largeron C. (éd.), Approches multiformes de la proximité, Hermès, Paris. 


\section{NOTES}

1. Pour les publications les plus récentes sur ce point, le lecteur peut se référer à Dupuy et Burmeister (2003) ou encore Pecqueur et Zimmermann (2004).

2. Ces auteurs définissent les ressources comme un potentiel latent de facteurs à exploiter, à organiser ou à révéler. Elles sont spécifiques lorsqu'elles ne peuvent être transférées.

3. L'apprentissage peut prendre une forme individuelle par l'inscription en mémoire d'expériences, mais en économie, il se fonde principalement sur les liens entre les agents (Johnson et Lundvall, 1992) : il nécessite donc beaucoup d'interactions pour permettre l'échange d'informations et la création de connaissances. C'est bien à l'apprentissage entendu comme une facette de la coordination que nous faisons référence ici.

4. Et ceci le distingue de la violence qui n'a pas besoin de justification. C'est seulement quand la contestation est possible, qu'il existe une alternative, que le pouvoir devient d'actualité.

5. Dans le cas qui nous occupe, ce niveau recouvre principalement le niveau national, parfois européen. Il est par contre plus rare que des acteurs entretiennent, au nom des pays, des relations avec des acteurs situés dans d'autres régions du monde.

6. Regroupant $40 \%$ de la population française et plus des deux tiers des communes (http://www.adcf.asso.fr).

7. Cf. Aubert et al. (2004) pour une classification des pays.

8. Ainsi, le pays de Haute-Gironde, dans la formulation de ses objectifs, insiste sur l'importance d'un équilibre à trouver entre un écosystème fragile, le développement économique et social et l'espace de vie des habitants. Adoptant une démarche éthique, la politique de logement, par exemple, devrait permettre de limiter les inégalités sociales et de mieux respecter l'environnement grâce à l'objectif de cohérence entre les politiques d'aménagement, d'équipement et d'habitat. Le pays de Gâtine (Poitou-Charentes) fait appel à une démarche participative et ascendante. Il a pour vocation de faire intervenir les habitants (élus, socioprofessionnels, associations) dans la définition des enjeux du territoire. Une commission de travail « prospective » a permis d'associer les différents acteurs du pays et les chambres consulaires, tandis que l'Université apporte une assistance technique. Ici, le principe d'interdisciplinarité fondant le développement durable est mis en œuvre, les acteurs fonctionnant sur le mode de la démocratie participative. Autre exemple : confronté à la pollution de sa principale réserve en eau, le pays de Beauce Gâtinais en Pithiverais a concentré ses efforts, dès sa création, sur la mise en place d'une politique de gestion globale de l'eau. Il s'agit de mettre en œuvre un véritable plan de sauvegarde destiné à réparer les errements du passé et à jeter les bases d'une gestion « durable » de la ressource, respectant de la sorte l'idée d'interdépendance temporelle entre les générations. 


\section{RÉSUMÉS}

La gouvernance locale peut-elle présenter des caractéristiques de durabilité ? Après être revenu sur le rôle ambigu de la proximité géographique, qui une fois associée aux proximités institutionnelle et organisationnelle, définit la gouvernance locale, nous nous interrogeons sur le caractère durable mais aussi non durable de celle-ci. Une illustration par les pays vient étayer l'analyse en démontrant que les principes fondateurs d'interdépendance et d'éthique du développement durable sont partiellement appliqués. Toutefois, le caractère durable des pays est limité par nombre de difficultés.

Can the local governance have characteristics of sustainability? After having reconsidered the ambiguous function of the geographical proximity, this article defines the local governance, understood like an association of geographical, institutional and organisational proximities. Moreover, this paper looks into the question of sustainable character of local governance. An illustration by the "pays" comes to support the analysis and shows that sustainable principles of interdependence and ethics are partially applied. However, this sustainable character is limited.

\section{INDEX}

Keywords : sustainable development, proximities, local governance

Mots-clés : développement durable, proximités, gouvernance locale, pays

\section{AUTEUR}

\section{DAMIEN TALBOT}

L'auteur est Maître de Conférences à l'Université Montesquieu-Bordeaux IV, au sein de l'IFReDE-GRES. Dans une perspective institutionnaliste, il cherche à expliquer la dynamique des coordinations productives entre acteurs à travers les processus d'apprentissage et de conflits. Et considérant que le lieu où prennent place les interactions entre acteurs compte autant que le moment durant lequel elles se déroulent, il fait appel aux concepts de proximité pour saisir le rôle de l'espace dans la dynamique des coordinations. De façon complémentaire, il a entrepris de développer la notion de gouvernance locale dans le but de contribuer encore à ce rapprochement et d'intégrer le rôle de l'acteur public dans sa réflexion. Il a récemment contribué à la rédaction d'un chapitre de l'ouvrage intitulé « Entreprises et Territoires : les nouveaux enjeux de la proximité », paru à la Documentation Française en 2003. 